\title{
Heart Failure with Preserved Ejection Fraction: A Review of the ACC/AHA Guidelines and Evidence-Based Management Strategy
}

Vikas Sunder, MD

\begin{abstract}
INTRODUCTION
Heart failure with preserved ejection fraction (HFpEF) is a clinical syndrome in which a patient has signs and symptoms of heart failure including dyspnea, fatigue, pulmonary rales, peripheral edema and an ejection fraction greater than 50\%. ${ }^{1-4}$ Approximately half of patients with heart failure have a preserved ejection fraction. ${ }^{2-5}$ HFpEF is considered a distinct clinical entity from other causes of heart failure with a preserved ejection fraction such as valvular heart disease, pericardial disease, and infiltrative cardiomyopathy. HFpEF carries a poor prognosis, with an annual mortality of $29 \%$ in patients discharged after an acute decompensated heart failure admission. ${ }^{5}$-year survival rates can be as poor as $50 \%{ }^{2}$ We know that patients with HFpEF are more likely to be older, female, and have hypertension. 3,4 Hypertension, in fact, is present in $80-90 \%$ of patients with HFpEF. ${ }^{3}$ The aim of this work is to summarize current understanding of HFpEF and review the latest ACC/AHA management guidelines while highlighting studies which provide the evidence for and against particular management strategies.
\end{abstract}

\section{PATHOPHYSIOLOGY}

The pathophysiologic mechanisms leading to HFpEF are complex. One proposed pathway involves understanding HFpEF within the context of a systemic pro-inflammatory state. In HFpEF, it is the high prevalence of co-morbid diseases such as obesity, hypertension, and diabetes mellitus which leads to a systemic pro-inflammatory state and causes coronary microvascular endothelial inflammation. ${ }^{6}$ This inflammation leads to low protein kinase $G$ activity in the cardiomyocytes leading to hypo-phosphorylation of titin favoring hypertrophy, high resting tension, and high diastolic left ventricular stiffness. ${ }^{6}$ Decreased nitric oxide bioavailability has also been implicated. ${ }^{6}$ Diastolic dysfunction can lead to elevated pulmonary venous pressure, congestion, and symptoms of heart failure. The presence of high diastolic filling pressure at rest or with exertion is a hallmark of HFpEF. ${ }^{2}$ Asymptomatic diastolic dysfunction without heart failure symptoms carries an increased mortality, but is a separate entity from HFpEF.? Heart failure with a reduced ejection fraction (HFrEF) is different in that it is driven by a loss of cardiomyocytes and is characterized by ventricular dilation. ${ }^{2.6}$ The presence of ventricular dilation is a key factor distinguishing HFrEF from HFpEF.?

\section{PHARMACOLOGIC MANAGEMENT}

There have been numerous advances in the pharmacologic management of HFrEF in the past 25 years related to blockade of the renin-angiotensinaldosterone system, beta blockade, neprilysin inhibition, and even ventricular assist devices. ${ }^{2,3}$ Unfortunately, most clinical trial data suggests that these modalities do not alter the course of HFpEF or provide a reduction in mortality. The 2013 ACC/AHA guideline for the management of heart failure gives a class I recommendation that systolic and diastolic blood pressure be controlled in patients with HFpEF. ${ }^{1}$ While control of hypertension is therefore considered a mainstay in management of HFpEF, there is currently no evidence that reducing the systemic blood pressure improves signs or symptoms of HFpEF. ACE inhibitors, ARBs, along with beta-blockers, may be used to treat hypertension in patients with HFpEF as a class Ila recommendation in the ACC/AHA guideline. Data from the ALLHAT trial showed that chlorthalidone could reduce the incidence of new-onset HFpEF compared with amlodipine and lisinopril, but this pertains to HFpEF prevention rather than treatment. ${ }^{8}$ There is no evidence in clinical studies that ACE inhibitors or ARBs improve morbidity or mortality in patients with HFpEF. The data is mixed with beta-blockers, with one recent metanalysis showing no cardiovascular or all-cause mortality benefit in patients with HFpEF. ${ }^{9}$ Regarding aldosterone antagonists, data from the TOPCAT trial showed that patients treated with spironolactone had fewer hospitalizations for heart failure as compared to patients treated with placebo, but that spironolactone did not reduce total deaths or hospitalizations from any cause during the study. ${ }^{10}$ The ACC/AHA gives a class IIb recommendation for the use of aldosterone antagonists like spironolactone to decrease hospitalizations in HFpEF.1 Statins have been shown to be beneficial in some studies. One Swedish study found that patients with HFPEF had a one-year survival of $85 \%$ when they were 
treated with statins versus $80 \%$ when not treated with statins. ${ }^{11}$ More recently, given that impairment in nitric oxide signaling has been implicated in the pathophysiology of HFpEF, as introduced above, investigators in the INDIE-HFpEF trial tested the use of inhaled inorganic nitrite delivered via a micro-nebulizer versus placebo over a 4-week period. The patients who received the inhaled inorganic nitrate did not demonstrate a statistically significant improved exercise capacity. ${ }^{12}$

\section{SYMPTOM-BASED MANAGEMENT AND QUALITY OF LIFE}

The only intervention which has consistently been shown to improve quality of life in patients with HFpEF as assessed by the Minnesota Living with Heart Failure Questionnaire is exercise. ${ }^{13}$ Referring patients to a cardiac rehabilitation program can improve exercise capacity. ${ }^{13}$ Weight loss can also be beneficial in obese patients as caloric restriction has already been shown to improve exercise capacity in patients with HFpEF.14 Maintenance of fluid balance is a pivotal part of symptom based management of HFpEF. The 2013 ACC/AHA guideline for the management of heart failure gives a class I recommendation that diuretics should be used for relief of volume overload in patients with HFpEF. ${ }^{1}$ This recommendation is based off findings from the CHAMPION trial in which patients had pulmonary artery monitors implanted to monitor their hemodynamics and providers made medication adjustments based on this primary data. ${ }^{15} \mathrm{~A}$ number of changes were made to diuretic medications and these patients had a reduction in heart failure related admissions at 6 months. ${ }^{15}$

\section{MANAGEMENT OF ATRIAL FIBRILLATION}

Atrial fibrillation is very common in patients with HFpEF, and occurs in approximately two-thirds of patients according to a community- based study in Olmstead County, Minnesota. ${ }^{16}$ Supraventricular arrhythmias like atrial fibrillation and associated tachycardia are dangerous for patients with HFpEF in that they impair atrial contraction and diastolic filling time. Patients with HFpEF and atrial fibrillation have an increased mortality. ${ }^{16}$ The 2013 ACC/AHA guideline recommends management of atrial fibrillation according to published clinical practice guidelines in patients with HFpEF which includes either a rate control or rhythm control strategy. ${ }^{1}$

\section{MANAGEMENT OF CORONARY ARTERY DISEASE}

Approximately two-thirds of patients with HFpEF have coronary artery disease demonstrated by coronary angiography. ${ }^{17}$ Patients with HFpEF and coronary artery disease have a higher mortality. ${ }^{17}$ Revascularization of all coronary stenoses greater than $50 \%$, that is, complete revascularization, may be associated with improved outcomes in these patients. One study found that patients with HFpEF and coronary disease who underwent complete revascularization had less reduction in ejection fraction at four-year follow-up echocardiography. Furthermore, patients who underwent complete re-vascularization by percutaneous intervention or bypass grafting survived longer than those patients who underwent incomplete revascularization or had no revascularization performed. ${ }^{17}$ Further prospective research is needed to determine optimal management of coronary artery disease in HFpEF. Patients with HFpEF who have angina can be treated symptomatically with beta-blockers or calcium channel blockers.

\section{CONCLUSION}

Optimal management of HFpEF remains unclear even as its prevalence and economic burden continue to increase. ${ }^{2-5}$ Further prospective trials enrolling this population of patients are needed. Patients with HFpEF represent a heterogenous group and there may not be a "one-size fit all" type of treatment, and this is likely not the best strategy. The latest ACC/AHA guideline for management of heart failure published in 2013 recommends symptomatic management with diuretics and control of co-morbid disease, including hypertension and atrial fibrillation. The evidence clearly supports the use of exercise in patients with HFpEF to both improve quality of life and increase exercise capacity. Further study is needed regarding the optimal amount and form of exercise which could be beneficial. Spironolactone may be used in patients with HFpEF to reduce hospitalizations related to heart failure.

\section{KEY POINTS}

1. The latest ACC/AHA guideline for management of heart failure provides a class I recommendation that the systolic and diastolic blood pressure be controlled in HFpEF and that diuretics be used for relief of volume overload.

2. Exercise is the only intervention which has been shown to improve quality of life in patients with HFpEF. Patients with HFpEF should be referred to a cardiac rehabilitation program.

3. Aldosterone antagonists may be considered to reduce future hospitalizations related to heart failure based on current evidence. 


\section{REFERENCES}

1. Yancy CW, Jessup M, Bozkurt B, et al. 2013 ACCF/AHA guideline for the management of heart failure: a report of the American College of Cardiology Foundation/American Heart Association Task Force on Practice Guidelines. Journal of the American College of Cardiology 62.16 (2013): e147-e239.

2. Reddy YNV and Borlaug BA. Heart failure with preserved ejection fraction. Current problems in cardiology 41.4 (2016): 145-188.

3. Andersen MJ and Borlaug BA. Heart failure with preserved ejection fraction: current understandings and challenges. Current cardiology reports 16.7 (2014): 501

4. Yancy CW, Lopatin M, Stevenson LW, et al. Clinical presentation, management and in-hospital outcomes of patients admitted with acute decompensated heart failure with preserved systolic function: a report from the Acute Decompensated Heart Failure National Registry (ADHERE) Database. Journal of the American College of Cardiology 47.1 (2006): 76-84.

5. Owan TE, Hodge DO, Herges RM, et al. Trends in prevalence and outcome of heart failure with preserved ejection fraction. New England Journal of Medicine 355.3 (2006): 251-259.

6. Paulus WJ and Tschöpe C. A novel paradigm for heart failure with preserved ejection fraction: comorbidities drive myocardial dysfunction and remodeling through coronary microvascular endothelial inflammation. Journal of the American College of Cardiology 62.4 (2013): 263-271.

7. Halley CM, Houghtailing P, Khalil KM, Thomas DJ, and Jaber W. Mortality rate in patients with diastolic dysfunction and normal systolic function. Archives of Internal Medicine 171.12 (2011): 1082-1087.

8. Davis RD, Kostis JB, Simpson LM, et al. Heart failure with preserved and reduced left ventricular ejection fraction in the antihypertensive and lipidlowering treatment to prevent heart attack trial. Circulation 118.22 (2008): 2259-2267.

9. Cleland JGF, Bunting KV, Flather MD, et al. Beta-blockers for heart failure with reduced, mid-range, and preserved ejection fraction: an individual patient-level analysis of double-blind randomized trials. European heart journal 39.1 (2017): 26-35.
10. Pitt B, Pfeffer MA, Assmann SF, et al. Spironolactone for heart failure with preserved ejection fraction. New England Journal of Medicine 370.15 (2014): 1383-1392.

11. Alehagen U, Benson L, Edner M, Dahlstrom U and Lund LH. Association Between Use of Statins and Mortality in Patients with Heart Failure and Ejection Fraction of Greater Than or Equal to 50\%. Circulation: Heart Failure (2015): CIRCHEARTFAILURE-115.

12. Borlaug BA, Anstrom KJ, Lewis GD, et al. Effect of Inorganic Nitrite vs Placebo on Exercise Capacity Among Patients With Heart Failure With Preserved Ejection Fraction: The INDIE-HFpEF Randomized Clinical Trial. JAMA 320.17 (2018): 1764-1773.

13. Pandey A, Parashar A, Kumbhani D, et al. Exercise training in patients with heart failure and preserved ejection fraction: a meta-analysis of randomized control trials. Circulation: Heart Failure (2014): CIRCHEARTFAILURE-114.

14. Kitzman DW, Brubaker P, Morgan T, et al. Effect of caloric restriction or aerobic exercise training on peak oxygen consumption and quality of life in obese older patients with heart failure with preserved ejection fraction: a randomized clinical trial. Jama 315.1 (2016): 36-46.

15. Abraham WT, Adamson PB, Bourge RC et al. Wireless pulmonary artery haemodynamic monitoring in chronic heart failure: a randomized controlled trial. The Lancet 377.9766 (2011): 658-666.

16. Zakeri R, Chamberlain AM, Roger VL, and Redfield MM. Temporal relationship and prognostic significance of atrial fibrillation in heart failure patients with preserved ejection fraction: a community-based study. Circulation (2013): CIRCULATIONAHA-113

17. Hwang SJ, Melenovsky V, and Borlaug BA. Implications of coronary artery disease in heart failure with preserved ejection fraction. Journal of the American College of Cardiology 63.25 Part A (2014): 2817-2827. 